\title{
Growing community networks with local events
}

\author{
Xin-Jian Xu ${ }^{a, b}$, Xun Zhang ${ }^{c}$, J. F. F. Mendes ${ }^{b}$ \\ ${ }^{a}$ Department of Mathematics, College of Science, Shanghai University, Shanghai \\ 200444, China \\ ${ }^{b}$ Departamento de Física da Universidade de Aveiro, 3810-193 Aveiro, Portugal \\ ${ }^{c}$ Centre for Computational Science and Engineering, National University of \\ Singapore, Singapore 117542
}

\begin{abstract}
The study of community networks has attracted considerable attention recently. In this paper, we propose an evolving community network model based on local processes, the addition of new nodes intra-community and new links intra- or intercommunity. Employing growth and preferential attachment mechanisms, we generate networks with a generalized power-law distribution of nodes' degrees.
\end{abstract}

Key words: Complex Networks; Community Networks

\section{Introduction}

Complex networks, evolved from the Erdös-Rényi random graph [1], are powerful models for describing many complex systems in biology, sociology, and technology [2]. In the past decade, the explosion of the general interest in the structure and the evolution of most real-world networks is mainly reflected in two striking characteristics. One is the small-world property [3], which suggests that a network has a highly degree of clustering like regular networks and a small average distance among any two nodes similar to random networks. The small-world phenomenon has been successfully described by network models with some degree of randomness [3,4]. The other is the scale-free behavior [5], which means a power-law distribution of connectivity, $P(k) \sim k^{-\gamma}$, where $P(k)$ is the probability that a node in the network has $k$ connections to other nodes and $\gamma$ is a positive real number determined by the given network. The origin of the scale-free behavior has been traced back to two mechanisms that are observed in many systems, growing and preferential attachment $[5,6]$. 
Recently, with the progress of research in networks, many other statistical characteristics of networks appeared on the stage. Of particular renown is the so-called "community" (or "modularity"). That is to say, a network is composed of many clusters of nodes, where the nodes in the same cluster are highly connected, while there are few links among the nodes belonging to different clusters. For instance, groups are formed in scientific collaboration networks [7]. Also, it has been found that dynamical processes on networks are affected by community structures, such as tendencies spread well within communities [8] and diffusion between different communities is slow [9].

In the study of community networks, most research has been directed in two distinct directions. On the one hand, attention has been paid to designing algorithms for detecting community structures in real networks. A pioneering method was made by Girvan and Newman [7], who introduced a quantitative measure for the quality of a partition of a network into communities. Later, a number of algorithms have been proposed in order to find a good optimization with the least computational cost. The fastest available procedures use greedy techniques [10] and extremal optimization [11], which are capable of detecting communities in large networks. On the other hand, research has focused on modeling of networks with community structures. In Ref. [12], a static social network was introduced where individuals belong to groups that in turn belong to groups of groups and so on. In Ref. [13], a networked seceder model was suggested to illustrate group formation in social networks. In Ref. [14], a growing bipartite network for social communities with group structures was proposed. Each of those models is constructed based on one aspect of reality.

In this paper, we introduce a network model with communities that gives a realistic description of local events $[15,16,17]$. The model incorporates three processes, the addition of new nodes intra-community and new links intraor inter-community. Using growing and preferential attachment mechanisms, we generate the community network with a good right-skewed distribution of nodes' degrees, which has been observed in many social systems.

\section{Model}

The Barabási-Albert network [5] only describes a particular type of evolving networks, the addition of new nodes preferential connecting to the nodes already present in the network. Systems in the real world, however, are much richer. For example, in scientific collaboration networks, a multidisciplinary scientist is not only collaborate with scientists in his research fields but also has a stronger desire to collaborate with scientists in other fields. In friendship networks, a person usually makes friends with people belonging to different communities besides the community he belongs to. To give a realistic descrip- 
tion of the network construction like that, we introduce a growing model of community networks based on local events, the addition of new nodes intracommunity and new links intra- or inter-community. The proposed model is defined as follows.

We start with $M(\geq 2)$ isolated communities and each community consists of a small number $n$ of isolated nodes. At each time step, we perform one of the following three operations.

(i) With probability $p$ we add a new node in a randomly chosen community. Here the randomly chosen means that the community is selected according to the uniform distribution. The new node is only connected to one node that already present in the selected community. We denote it as the $u$ th commnuity. The probability that node $i$ in community $u$ will be selected is proportional to its intra-community degree

$$
\prod\left(k_{i}^{\text {intra }}\right)=\frac{k_{u, i}^{\text {intra }}+1}{\sum_{j}\left(k_{u, j}^{\text {intra }}+1\right)},
$$

where the sum runs over nodes in community $u$ and $k_{u, i}^{\text {intra }}$ is the intra-community degree of node $i$ in community $u$.

(ii) With probability $q$ we add a new link in a randomly chosen community. For this we randomly select a node in a randomly chosen community $u$ as the starting point of the new link. The other end of the link is selected in the same community with the probability given by Eq. (1).

(iii) With probability $r(=1-p-q)$ we add a new link between two communities. For this we randomly select a node in a randomly chosen community $u$ as the starting point of the new link. The other end $i$ of the link selected in the other community $v$ is proportional to its inter-community degree

$$
\prod\left(k_{i}^{\text {inter }}\right)=\frac{k_{v, i}^{\text {inter }}+1}{\sum_{v \neq u ; j}\left(k_{v, j}^{\text {inter }}+1\right)},
$$

where the sum runs over nodes in all communities except for community $u$ and $k_{v, i}^{\text {inter }}$ is the inter-community degree of node $i$ in community $v$.

After $t$ time steps, this scheme generates a network of $M n+p t$ nodes and $t$ links. The parameters $p, q$, and $r$ control the network structure. In the case of small $r$, the generated network will have a strong community structure. Notice that whatever process is chosen in the network growth, only one link is added to the system at each time step (duplicate and self-connected edges are forbidden), however, this is not essential. We choose link probabilities $\prod\left(k_{i}^{\text {intra }}\right)$ and $\Pi\left(k_{i}^{\text {inter }}\right)$ to be proportional to $k_{i}^{\text {intra }}+1$ and $k_{i}^{\text {inter }}+1$, respectively, such that there is a nonzero probability of isolated nodes acquiring new links. 


\section{Degree distribution}

In our community network, the degree of a node consists of two parts, the intracommunity degree and the inter-community degree. Increase in the node's connectivity can be divided into two processes, the increases of the intracommunity degree and the inter-community degree. In each process, we assume that $k_{i}^{\text {intra }}$ and $k_{i}^{\text {inter }}$ change continuously, and the probabilities $\prod\left(k_{i}^{\text {intra }}\right)$ and $\Pi\left(k_{i}^{\text {inter }}\right)$ can be interpreted as the rates at which $k_{i}^{\text {intra }}$ and $k_{i}^{\text {inter }}$ change, respectively. Thus, the operations (i)-(iii) all contribute to $k_{i}$, each being incorporated in the continuum theory as follows.

(i) Addition of a new node in a randomly chosen community with probability $p$ :

$$
\frac{\partial k_{u, i}^{\text {intra }}}{\partial t}=p \frac{1}{M} \frac{k_{u, i}^{\text {intra }}+1}{\sum_{j}\left(k_{u, j}^{\text {intra }}+1\right)} .
$$

(ii) Addition of a new link in a randomly chosen community with probability $q$ :

$$
\frac{\partial k_{u, i}^{\text {intra }}}{\partial t}=q\left[\frac{1}{N}+\frac{1}{M} \frac{k_{u, i}^{\text {intra }}+1}{\sum_{j}\left(k_{u, j}^{\text {intra }}+1\right)}\right],
$$

where $N$ is the number of total nodes. The first term on the right-hand side (rhs) corresponds to the random selection of one end of the new link, while the second term on the rhs reflects the preferential attachment (Eq. (1)) used to select the other end of the link.

(iii) Addition of a new links between two communities with probability $r$ :

$$
\frac{\partial k_{v, i}^{\text {inter }}}{\partial t}=r\left[\frac{1}{N}+\left(1-\frac{1}{M}\right) \frac{k_{v, i}^{\text {inter }}+1}{\sum_{v \neq u ; j}\left(k_{v, j}^{\text {inter }}+1\right)}\right] .
$$

The first term on the rhs represents the random selection of one end of the new link, while the second term on the rhs considers the preferential attachment (Eq. (2)) used to select the other end of the link in the other community.

Combing the contribution of above processes, we have

$$
\begin{aligned}
& \frac{\partial k_{u, i}^{\text {intra }}}{\partial t}=\frac{p+q}{M} \frac{k_{u, i}^{\text {intra }}+1}{\sum_{j}\left(k_{u, j}^{\text {intra }}+1\right)}+\frac{q}{N}, \\
& \frac{\partial k_{v, i}^{\text {inter }}}{\partial t}=\frac{r}{N}+r \frac{M-1}{M} \frac{k_{v, i}^{\text {inter }}+1}{\sum_{v \neq u, j}\left(k_{v, j}^{\text {inter }}+1\right)},
\end{aligned}
$$

with 


$$
\begin{aligned}
\sum_{j}\left(k_{u, j}^{\text {intra }}+1\right) & =\sum_{j} k_{u, j}^{\text {intra }}+\frac{N}{M} \\
& =2 t\left(p \frac{1}{M}+q \frac{1}{M}\right)+\frac{M n+p t}{M} \\
& =\frac{3 p+2 q}{M} t+n, \\
\sum_{v \neq u ; j}\left(k_{v, j}^{\text {inter }}+1\right) & =\sum_{v \neq u ; j} k_{v, j}^{\text {inter }}+N\left(1-\frac{1}{M}\right) \\
& =2 t r \frac{M-1}{M}+(M n+p t) \frac{M-1}{M} \\
& =\frac{(2-p-2 q)(M-1)}{M} t+(M-1) n .
\end{aligned}
$$

We can simplify Eqs. (6) and (7) for large $t$

$$
\begin{aligned}
& \frac{\partial k_{u, i}^{\text {intra }}}{\partial t} \approx \frac{p+q}{3 p+2 q} \frac{\left(k_{u, i}^{\text {intra }}+1\right)}{t}+\frac{q}{p t} \\
& \frac{\partial k_{v, i}^{\text {inter }}}{\partial t} \approx \frac{1-p-q}{2-p-2 q} \frac{\left(k_{v, i}^{\text {inter }}+1\right)}{t}+\frac{1-p-q}{p t} .
\end{aligned}
$$

The boundary conditions of the intra-community degree and the inter-community degree at initial time $t_{s}$ can be estimated in the sense of mathematical expectations, $k_{u, i}^{\text {intra }}\left(t_{s}\right)=p+q$ and $k_{v, i}^{\text {inter }}\left(t_{s}\right)=r$, respectively. So we write the solutions of Eqs. (8) and (9)

$$
\begin{aligned}
& k_{u, i}^{\text {intra }}(t)=\frac{p^{3}+p^{2}+2 p^{2} q+4 p q+p q^{2}+2 q^{2}}{p(p+q)}\left(\frac{t}{t_{s}}\right)^{\frac{p+q}{3 p+2 q}}-\frac{p^{2}+4 p q+2 q^{2}}{p(p+q)}(10) \\
& k_{v, i}^{\text {inter }}(t)=\frac{2-2 q-p q+p-p^{2}}{p}\left(\frac{t}{t_{s}}\right)^{\frac{1-p-q}{2-p-2 q}}-\frac{2-2 q}{p}
\end{aligned}
$$

In random networks, the degree distribution can be calculated by

$$
P(k)=\frac{1}{t} \sum_{i=1}^{t} \delta\left(k_{i}(t)-k\right),
$$

which gives 

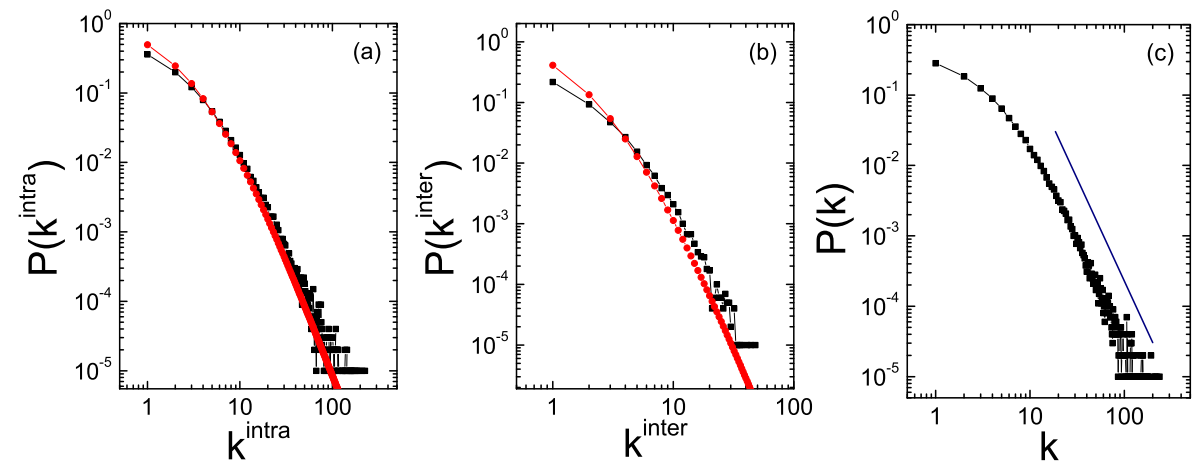

Fig. 1. (Color online) Log-log representation of distributions of intra-community degree (a), inter-community degree (b), and total degree (c) of nodes. All the simulation results (squares) display good right-skewed distributions. The circles in (a) and (b) denote analytical results predicted by Eqs. (13) and (14), respectively. The solid line in (c) is guide to the eye with power-law decay exponent $\gamma=3.0$. The experiment network has a total number of nodes $N=10^{5}$ with parameters $M=10$, $n=5, p=0.4$, and $q=0.4$, respectively.

$$
\begin{aligned}
P\left(k^{\text {intra }}\right) & =\frac{3 p^{2}+2 p q}{p^{2}+2 q^{2}+4 p q+2 p^{2} q+p q^{2}+p^{3}} \\
& \times\left[\frac{p^{2}+4 p q+2 q^{2}+\left(p^{2}+p q\right) k^{\text {intra }}}{p^{2}+2 q^{2}+4 p q+2 p^{2} q+p q^{2}+p^{3}}\right]^{-\left(3+\frac{p}{p+q}\right)} \\
P\left(k^{\text {inter }}\right) & =\frac{2 p-2 p q-p^{2}}{2-p-4 q-2 p^{2}+2 q^{2}+2 p^{2} q+p q^{2}+p^{3}} \\
& \times\left[\frac{2-2 q+p k^{\text {inter }}}{2+p-2 q-p q-p^{2}}\right]^{-\left(3+\frac{p}{1-p-q}\right)}
\end{aligned}
$$

Thus, the degree distribution of our network obeys a generalized power-law form

$$
P(k) \sim[A(p, q) k+B(p, q)]^{-\gamma(p, q)}
$$

In Fig. 1 we present numerical results of distributions of the intra-community degree, the inter-community degree, and the total degree of nodes in log-log scale. The experimental network is generated by the proposed scheme with $N=10^{5}, M=10, n=5, p=0.4$, and $q=0.4$, respectively. The distributions of the intra-community degree and the inter-community degree, shown in Figs. 1(a) and 1(b), agree with analytical results of Eqs. (13) and (14), respectively. The small deviations between computer simulations and analytical solutions at both ends of the distributions appears to be the mathematical approximation of the boundary conditions and the finite size effect due to the relatively small network sizes used in the simulations. According to the evolving rule of our 


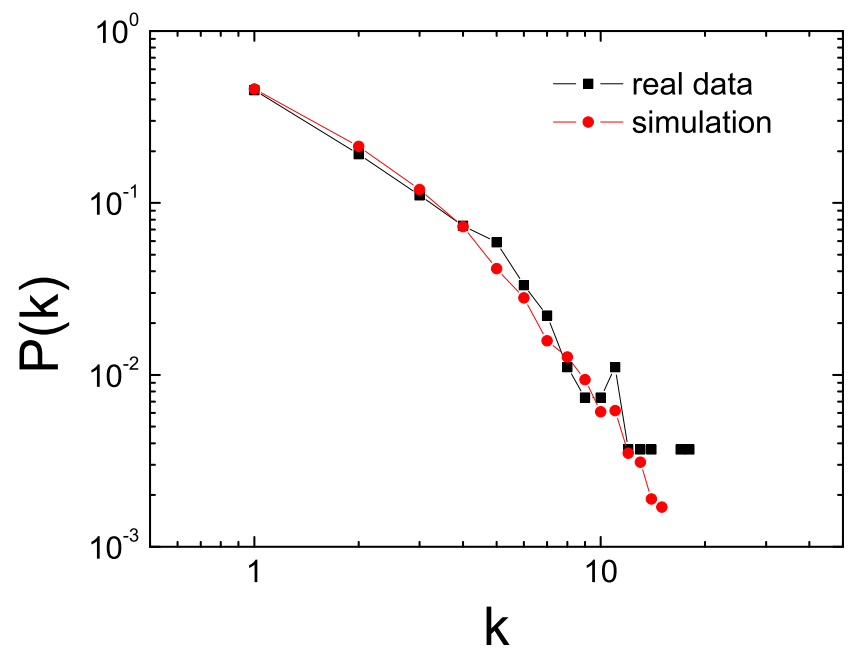

Fig. 2. (Color online) The degree distribution of econophysicists (squares) of an econophysics scientific collaboration network [19]. The circles correspond to computer simulations of our model with parameters $M=10, n=2, p=0.4$, and $q=0.4$, respectively.

network, nodes with larger intra- (or inter-) degree have higher probability to gain new links, then the usual degree preferential attachment is reasonably kept. This means that the right-skewed character of the network, such as the node's total degree, will retain. As shown in Fig. 1(c), the total degree distribution of nodes is well expected showing a good right-skewed character, which is reasonably in agreement with the condition of many realistic systems [18].

To illustrate the predictive power, we also compare the numerical result of our network with the statistics of an econophysics collaboration network. In the econophysics collaboration network, each node represents one scientist. If two scientists have collaborated one or more papers, they would be connected by an edge. Zhang et al. took the largest connected component of this network, which includes 271 nodes and 371 edges, and provided the best division, i.e., $M=10$ [19]. In Fig. 2 we plot the degree distribution of econophysicists of the econophysics collaboration network which is fitted by computer simulations of our network starting with 10 communities. To gain $p$ and $q$, we fit the connectivity distribution $P(k)$ obtained from this collaboration network with Eq. (15), obtaining a good overlap for $p=0.75$ and $q=0.15$ (Fig. 2). 


\section{Conclusion}

Networks with community structures underlie many natural and artificial systems. It is becoming essential to model and study this kind topological feature. We presented a simplified mechanism for networks organized in communities, which corresponds to local events during the system's growth. The generated network is highly clustered and has a good rightskewed distribution of connectivity, which have been found very common in most realistic systems. The present paper only suggests a simple way for generating community networks. The shape of the resulting network is deterministic in some extent. It is more interesting to model the evolution of communities, especially the self organization (or emergence) of communities in the natural world [20], e.g., expansion and shrinkage, which is left to future work.

\section{Acknowledgements}

The authors acknowledge financial support from NSFC/10805033, SocialNets/217141, STCSM/08ZR1408000, PTDC/FIS/71551/2006, and FCT/SFRH/BPD/30425/2006.

\section{References}

[1] P. Erdös, A. Rényi, Publ. Math. 6 (1959) 290.

[2] R. Albert, A.-L. Barabási, Rev. Modern Phys. 74 (2002) 47; S.N. Dorogovtsev, A.V. Goltsev, J.F.F. Mendes, Rev. Modern Phys. 80 (2008) 1275.

[3] D. J. Watts, S. H. Strogatz, Nature 393 (1998) 440.

[4] M. E. J. Newman, D. J. Watts, Phys. Rev. E 60 (1999) 7332.

[5] A.-L. Barabási, R. Albert, Science 286 (1999) 509.

[6] P. L. Krapivsky, S. Redner, F. Leyvraz, Phys. Rev. Lett. 85 (2000) 4629.

[7] M. Girvan, M. E. J. Newman, Proc. Natl. Acad. Sci. USA 99 (2002) 7821.

[8] L. M. A. Bettencourt, arXiv:cond-mat/0304321.

[9] K. A. Eriksen, I. Simonsen, S. Maslov, K. Sneppen, Phys. Rev. Lett. 90 (2003) 148701.

[10] M. E. J. Newman, Phys. Rev. E 69 (2004) 066133.

[11] J. Duch, A. Arenas, Phys. Rev. E 72 (2005) 027104. 
[12] D. J. Watts, P. S. Dodds, M. E. J. Newman, Science 296 (2002) 1302.

[13] A. Grönlund, P. Holme, Phys. Rev. E 70 (2004) 036108.

[14] J. D. Noh, H.-C. Jeong, Y.-Y. Ahn, H. Jeong, Phys. Rev. E 71 (2005) 036131.

[15] R. Albert, A.-L. Barabási, Phys. Rev. Lett. 85 (2000) 5234.

[16] S. N. Dorogovtsev, J. F. F. Mendes, Europhys. Lett. 52 (2000) 33.

[17] X. Li, G. Chen, Physica A, 328 (2003) 274.

[18] L. A. N. Amaral, A. Scala, M. Barthelemy, H. E. Stanley, Proc. Natl. Acad. Sci. USA 97 (2000) 11149; M. E. J. Newman, Proc. Natl. Acad. Sci. USA 98 (2001) 404.

[19] P. Zhang, M. Li, J. Wu, Z. Di, Y. Fan, Physica A 367 (2006) 577.

[20] J. M. Kumpula, J. P. Onnela, J. Saramäki, K. Kaski, J. Kertész, Phys. Rev. Lett. 99 (2007) 228701. 